\title{
Critical implication of the PTEN/PI3K/AKT pathway during BMP2-induced heterotopic ossification
}

\author{
JUN DONG, XIQIANG XU, QINGYU ZHANG, ZENONG YUAN and BINGYI TAN \\ Department of Orthopaedics, Shandong Provincial Hospital, \\ Affiliated to Shandong First Medical University, Jinan, Shandong 250021, P.R. China
}

Received July 20, 2020; Accepted January 8, 2021

DOI: $10.3892 / \mathrm{mmr} .2021 .11893$

\begin{abstract}
Heterotopic ossification (HO) is characterized by extraskeletal ossification in soft tissue. Thus far, there is a lack of effective drug therapy against HO. Loss of PTEN in osteoblasts has been reported to accumulate bone mass in skeletal development and promote fracture healing in association with the activation of the PI3K/AKT pathway. However, the role of the PTEN/PI3K/AKT signaling in $\mathrm{HO}$ pathogenesis remains unknown. The present study investigated the implication of this pathway during BMP2-induced osteogenic differentiation and ectopic bone formation. It was shown that overexpression of PTEN inhibited proliferation but stimulated apoptosis in mesenchymal pluripotent $\mathrm{C} 3 \mathrm{H} 10 \mathrm{~T} 1 / 2$ cells. PTEN also inhibited BMP2-induced osteoblast differentiation, whereas BMP2 repressed PTEN expression and subsequently activated PI3K/AKT. The PI3K inhibitor, LY294002, blocked BMP2-induced osteoblastogenesis, suggesting that the $\mathrm{PI} 3 \mathrm{~K} / \mathrm{AKT}$ pathway is critically required for BMP2 to initiate osteoblastogenesis. In vivo, implantation of BMP2 in muscle induced ectopic endochondral ossification. Strikingly, this bone-forming capacity was notably suppressed by the PI3K inhibitor LY294002. Hence, the results of the present study demonstrated that the PI3K/AKT signaling activity is indispensable for BMP2 to induce ectopic new bone. Targeting the PI3K/AKT pathway using inhibitor(s) may represent a potential molecular therapy for the treatment against HO.
\end{abstract}

Correspondence to: Dr Bingyi Tan, Department of Orthopaedics, Shandong Provincial Hospital, Affiliated to Shandong First Medical University, 324 The Five Weft Seven Road, Jinan, Shandong 250021, P.R. China

E-mail: bingytan07@gmail.com

Abbreviations: ALP, alkaline phosphatase; BMP, bone morphogenetic protein; H\&E, hematoxylin and eosin; MSC, mesenchymal stem cells; PI3K, phosphatidylinositol 3-kinase; PIP2, phosphatidylinositol (4,5)-bisphosphate; PIP3, phosphatidylinositol $(3,4,5)$-triphosphate; PTEN, phosphatase and tensin homolog deleted from chromosome 10; RT-qPCR, reverse transcription-quantitative PCR; SO, Safranin O

Key words: PTEN, PI3K/AKT, heterotopic ossification, osteoblast differentiation

\section{Introduction}

Clinically, heterotopic ossification (HO) is a pathologic process defined as the formation of ectopic bone in muscle and soft tissues. HO has long been described as a congenital or inherited malformation, or a complication of various conditions, such as paraplegia, traumatic injury, post hip arthroplasty, as well as ossification of the posterior longitudinal ligament (OPLL) (1). The histopathology of HO is well investigated; however, the molecular mechanism that regulates the pathogenesis of extraskeletal ossification remains largely unknown. Recent data have suggested that the bone morphogenetic protein (BMP) family may play a central role in $\mathrm{HO}$ development. For example, the expression of BMP2, BMP4, BMP7 and BMP9 was substantially elevated in spinal cord injury-induced $\mathrm{HO}$ (2). HO formation also frequently occurred in patients with osteonecrosis of femoral head (ONFH) receiving intraoperative recombinant human BMP2 protein (3). There is currently a lack of effective drug therapy against HO. It is imperative to explore the signaling pathway underlying the development of ectopic bone formation to develop novel molecular therapy for this disease.

As a tumor suppressor, the phosphatase and tensin homolog deleted from chromosome 10 (PTEN) functions primarily via dephosphorylation of phosphatidylinositol $(3,4,5)$-triphosphate $\left(\mathrm{PIP}_{3}\right)$ to phosphatidylinositol $(4,5)$-bisphosphate $\left(\mathrm{PIP}_{2}\right)$, leading to the negative regulation of the phosphatidylinositol 3-kinase (PI3K)/AKT activity (4). The PTEN/PI3K/AKT nexus has been implicated in diverse physiological and pathological conditions and plays a crucial role in the regulation of cell growth, apoptosis, metabolism and other processes (5). This signaling axis is also involved in embryonic bone development and fracture healing. Loss of PTEN in mature osteoblasts leads to profoundly accumulated postnatal bone mass at all skeletal sites and notably decreased apoptosis (6). The knockout of PTEN in osteoblasts also enhances intramembranous and late endochondral fracture repair (7). Recently, micro (mi)RNA-26a-5p has been shown to inhibit PTEN expression and promote the bone-healing process in fracture patients with traumatic brain injury (8). Being the direct target of PTEN, PI3K/AKT is also involved in osteoblast function and bone development. The whole-body deletion of AKT1 causes decreased bone mass, which associates with enhanced apoptosis and impaired osteoblast differentiation (9). PI3K is 
required for BMP2 to induce osteoblast differentiation (10). Interestingly, an inverse association between BMP2 and PTEN has been reported, in which BMP2 could downregulate PTEN expression at the protein level, an effect mediated via the RAS/ERK signaling pathway (11).

Although the PTEN/PI3K/AKT pathway is critically involved in skeletogenesis and fracture healing, it remains unknown whether this pathway impacts HO. In the present study, the mouse mesenchymal stem cell line $\mathrm{C} 3 \mathrm{H} 10 \mathrm{~T} 1 / 2$ were cultured and treated with recombinant human BMP2 (rhBMP2) to induce osteoblast differentiation. An ectopic ossification model was also established by implantation of rhBMP2 into the muscle of mice. It was hypothesized that PTEN inhibits BMP2-induced osteoblast differentiation, whereas BMP2 activates PI3K/AKT via its inhibition of PTEN. It was also hypothesized that PI3K/AKT signaling activity is indispensable for BMP2 to induce osteogenic differentiation in vitro and ectopic bone formation in vivo.

\section{Materials and methods}

Cell culture and stable transfection. Mouse fibroblast-like pluripotent stem cell line C3H10T1/2 (clone 8; American Type Culture Collection) was used in the present study; this cell line undergoes osteoblast lineage commitment, early and late osteoblast differentiation, and osteoblast mineralization in response to osteogenic agents (such as BMP2), thus providing an ideal model for osteoblast-associated studies in the literature (12-15).

C3H10T1/2 cells were cultured at $37^{\circ} \mathrm{C}$ in humidified air with $5 \% \mathrm{CO}_{2}$ in a growth medium containing Dulbecco's $\mu$ odified Eagle's $\mu$ edium (DMEM; Invitrogen; Thermo Fisher Scientific, Inc.) with 10\% fetal calf serum (FCS; Invitrogen; Thermo Fisher Scientific, Inc.). Cells were authenticated using small tandem repeat analysis and routinely tested to assure no mycoplasma contamination. For stable transfection, the human PTEN gene was subcloned into the pcDNA3.1 plasmid vector (Invitrogen; Thermo Fisher Scientific, Inc.). The pcDNA-PTEN was transfected into C3H10T1/2 cells using Lipofectamine ${ }^{\circledR} 2000$ transfection reagent (Invitrogen; Thermo Fisher Scientific, Inc.) following the manufacturer's protocol. After transfection, cells were treated with G418 at a dosage of $800 \mu \mathrm{g} / \mathrm{ml}$ at $37^{\circ} \mathrm{C}$ for 10 days to select for positive clones. Individual colonies were pooled together and transferred to a new culture dish. After selection, stable cell lines overexpressing PTEN were cultured in DMEM-10\% FCS at $37^{\circ} \mathrm{C}$. Cells transfected with pcDNA3.1 alone were used as controls.

Proliferation and apoptosis assay. The proliferation of C3H10T1/2 cells was evaluated using an MTT Proliferation kit (Roche Applied Science). Cells were plated in 96-well plates at a density of $5 \times 10^{3}$ cells/well in $100 \mu 1$ medium. After $24 \mathrm{~h}$, cells were incubated with $10 \mu \mathrm{l}$ MTT reagent for $2 \mathrm{~h}$. Then, $100 \mu \mathrm{l}$ of the solubilization solution from the kit was added to dissolve the formazan product. Absorbances at $570 \mathrm{~nm}$ were measured.

For the apoptosis assay, $\mathrm{C} 3 \mathrm{H} 10 \mathrm{~T} 1 / 2$ cells were cultured under serum-starved conditions (DMEM-0.5\% FCS) for $24 \mathrm{~h}$. The proapoptotic activity was determined by assessing the caspase-3 activity using the Caspase-3 Colorimetric Assay kit (R\&D Systems, Inc.) according to the manufacturer's protocol. Absorbances at $405 \mathrm{~nm}$ were read on a microplate reader.

Reverse transcription-quantitative PCR (RT-qPCR). For RT-qPCR analysis, total RNA was isolated from cells using the TRIzol reagent (Invitrogen; Thermo Fisher Scientific, Inc.). RNA $(2 \mu \mathrm{g})$ was reverse transcribed to $\mathrm{cDNA}$ at $50^{\circ} \mathrm{C}$ for 50 min using SuperScript III Reverse Transcriptase (Thermo Fisher Scientific, Inc.). PCR reactions were performed with SYBR green master mix (Thermo Fisher Scientific, Inc.). The cycling conditions were: $50^{\circ} \mathrm{C}$ for $2 \mathrm{~min} ; 95^{\circ} \mathrm{C}$ for $10 \mathrm{~min}$; then $95^{\circ} \mathrm{C}$ for $15 \mathrm{sec}$ and $60^{\circ} \mathrm{C}$ for $1 \mathrm{~min}$ for 40 cycles. The PCR primers for the mouse genes are listed in Table I. PCR products were analyzed with the ABI PRISM 7900HT Sequence Detections System (Applied Biosystems; Thermo Fisher Scientific, Inc.). To ensure primer specificity, one dissociation stage was added to produce the melting curve at the end of the cycling condition. The relative mRNA concentrations of the target genes were determined with ABI software (RQ Manager; Version 1.2), which normalizes the target gene threshold cycle to that of endogenous B2M transcripts $(\Delta \Delta \mathrm{Ct})$, using the formula $2^{-\Delta \Delta \mathrm{Cq}}$ to define fold change (16).

Western blotting. Cell lysates were prepared using RIPA Lysis buffer (Sigma-Aldrich; Merck KGaA). The samples (25 $\mu \mathrm{g})$ were electrophoresed on a $10 \%$ SDS polyacrylamide gel (Invitrogen; Thermo Fisher Scientific,Inc.) and were transferred onto a polyvinylidene difluoride membrane (EMD Millipore). Membranes were washed with TBS/0.1\% Tween-20 (TBST) and blocked with $1 \%$ BSA in TBST for $1 \mathrm{~h}$ at room temperature. Primary antibodies (Table II) were added to the membranes overnight at $4^{\circ} \mathrm{C}$. Blots were washed three times with TBST, followed by incubation with horseradish peroxidase-conjugated secondary antibodies (Bio-Rad Laboratories, Inc.) for $1 \mathrm{~h}$ at room temperature. After washing with TBST, the membrane was detected with ECL Detection reagents (Thermo Fisher Scientific, Inc.) and exposed to X-ray film. Densitometry of the protein bands was analyzed using ImageJ software (v1.51j8; National Institutes of Health).

Osteogenic differentiation. Confluent C3H10T1/2 cells were incubated in osteogenic medium containing DMEM-10\% FCS, $50 \mu \mathrm{g} / \mathrm{ml}$ ascorbic acid, $10 \mathrm{mM}$ sodium $\beta$-glycerophosphate, and $200 \mathrm{ng} / \mathrm{ml}$ recombinant human BMP2 (rhBMP2; Sigma-Aldrich; Merck KGaA). The osteogenic medium was replaced every $48 \mathrm{~h}$. The activity of alkaline phosphatase (ALP) was examined on day 3 using a colorimetric assay kit (Abcam). This kit used p-nitrophenyl phosphate as a phosphatase substrate that turned yellow at OD 405 when dephosphorylated by ALP. For ALP staining, cells were washed with PBS, fixed with $70 \%$ ethanol for $10 \mathrm{~min}$ at room temperature, and incubated with NBT/BCIP solution for $20 \mathrm{~min}$ at room temperature. The positively stained cells were monitored using a light microscope (x200; Olympus). The secretion of osteocalcin was examined using a mouse ELISA kit (cat. no. MBS2020904; MyBioSource, Inc.) after cells were cultured for 7 days. Cells were stained using the von Kossa method for calcium deposition at room temperature after 2 weeks. Briefly, cells were fixed with $4 \%$ 
Table I. PCR primers.

\begin{tabular}{lll}
\hline Gene & \multicolumn{1}{c}{ Forward, 5'-3' } & \multicolumn{1}{c}{ Reverse, 5'-3' } \\
\hline Alpl & GTTGCCAAGCTGGGAAGAACAC & CCCACCCCGCTATTCCAAAC \\
Collal & CCTGCCTGCTTCGTGTAA & GGTCACGTTCAGTTGGTCA \\
Run $x$ 2 & GGGAGACAACAGGGAGGAAAC & CAGGCTTCCTGCCAGTACCT \\
Sp7 & CGGTCTCCTTCCAGGATGGT & GCTTCCGTCAGCGTCAACA \\
Pten & AGCGACCACTTGAGCAAACAT & GCGGCTGATTGGCTTCTTCT \\
B $2 \mathrm{M}$ & AATTCCCAGTCAGAGGCGCTATGT & GATTGCAAGTTCCGCCACTGAACA \\
& AAATGCTGAAGAACGGGAAA & GATGCTTGATCACATGTCTCG
\end{tabular}

Table II. Antibodies used.

\begin{tabular}{|c|c|c|c|}
\hline Antibody & Dilution & Manufacturer & Cat. no. \\
\hline PTEN & $1: 500$ & Santa Cruz Biotechnology, Inc. & SC-7974 \\
\hline Phospho-AKT & $1: 1,000$ & Cell Signaling Technology, Inc. & 9271 \\
\hline AKT & $1: 1,000$ & Cell Signaling Technology, Inc. & 9272 \\
\hline$\beta$-actin & $1: 1,000$ & Cell Signaling Technology, Inc. & 4967 \\
\hline Anti-mouse IgG & $1: 3,000$ & Cell Signaling Technology, Inc. & 7076 \\
\hline Anti-rabbit IgG & $1: 3,000$ & Cell Signaling Technology, Inc. & 7074 \\
\hline
\end{tabular}

paraformaldehyde for $20 \mathrm{~min}$ and washed with water twice. Cells were then treated with $1 \%$ silver nitrate solution under ultraviolet light for $30 \mathrm{~min}$. After washing with distilled water, cells were incubated with $5 \%$ sodium thiosulfate for 5 min and were counterstained with $1 \%$ nuclear fast red solution for $5 \mathrm{~min}$. Cells were also stained with Alizarin Red for mineralization at room temperature after being cultured in the osteogenic medium after 4 weeks. In brief, cells were fixed with $4 \%$ paraformaldehyde for 20 min and washed with water. Cells were then treated with $2 \%$ Alizarin Red S solution for $5 \mathrm{~min}$. The quantification of stains was performed using the ARed-Q assay (cat. no. 8678, ScienCell). Following von Kossa and Alizarin Red S staining, cells were monitored using a light microscope (magnification, x200; Olympus Corporation). The expression of Runx 2 and osterix (the two key transcription factors for osteoblasts), ALP (early osteoblast differentiation marker), collagen type I (COL1A1), and osteocalcin (late osteoblast differentiation marker) was examined using qPCR analysis.

Ectopic bone-formation model. A total of 18 male BALB/c mice (12-14 weeks old) were utilized for the present study. All animal procedures were reviewed and approved by the Institutional Animal Care and Use Committee of the Shandong Provincial Hospital (approval no. M2018143). Mice were anesthetized using an intraperitoneal injection of a cocktail of ketamine $(80 \mathrm{mg} / \mathrm{kg}) / x y l a z i n e ~(10 \mathrm{mg} / \mathrm{kg})$. A 5-mm skin incision was made on the dorsal surface of the right lower limb; then a muscle pouch was created in the gluteus of mice. Freeze-dried absorbable collagen sponges $(5 \times 5 \times 5 \mathrm{~mm}$, Helistat) were mixed with $20 \mu \mathrm{g}$ rhBMP2 and placed into the muscle pouch. The incision was closed with sutures. Animals were subcutaneously injected with an analgesic agent, buprenorphine, at a dosage of $0.1 \mathrm{mg} / \mathrm{kg}$ twice a day for 3 days. Following the operation, animals were divided into two groups (9 mice/each). One group of mice were injected intraperitoneally with PI3K inhibitor LY294002 (Calbiochem) at a dosage of $25 \mathrm{mg} / \mathrm{kg}$ immediately and twice weekly. Other animals were injected with the same volume of DMSO (vehicle) as controls. For radiography, animals were examined for ossification using a Faxitron MX20 X-ray system (Faxitron Bioptics) at 3 weeks following the operation. Animals were monitored daily for their health and behaviors. All mice were in good health and survived well after implantation of rhBMP2.

For histological analysis, mice were sacrificed using standard $\mathrm{CO}_{2}$ euthanasia at 9 days and 3 weeks, respectively, after the operation. A fill rate of $30 \%$ of the chamber volume/min of $\mathrm{C}_{\mathrm{O} 2}$ to the existing air in the chamber was used for the animals to achieve fast unconsciousness with minimal distress. The death of mice was confirmed by ascertaining cardiac and respiratory arrest.

Tissue samples were harvested, fixed in $4 \%$ paraformaldehyde at $4^{\circ} \mathrm{C}$ for 2 days, decalcified in 20\% EDTA (pH 7.4) at $4^{\circ} \mathrm{C}$ for 10 days, and embedded in paraffin. Sections $(7 \mu \mathrm{m})$ were stained with Safranin O (SO) and hematoxylin and eosin (H\&E). For SO staining, deparaffinized and rehydrated sections were successively incubated with $0.05 \%$ fast green solution for $5 \mathrm{~min}, 1 \%$ acetic acid solution for $30 \mathrm{sec}$, and $0.1 \% \mathrm{SO}$ solution for $5 \mathrm{~min}$ at room temperature. For $\mathrm{HE}$ staining, sections were incubated with hematoxylin solution for $3 \mathrm{~min}$ and eosin solution for $1 \mathrm{~min}$ at room temperature. Bone histomorphometric analysis was performed as previously reported (17). An analysis image window measuring $2 \mathrm{~mm}^{2}$ was established for evaluation of bone volume as a percentage of total tissue volume (BV/TV \%), trabecular thickness $(\mu \mathrm{m})$, trabecular number (per mm), and trabecular separation $(\mu \mathrm{m})$. 

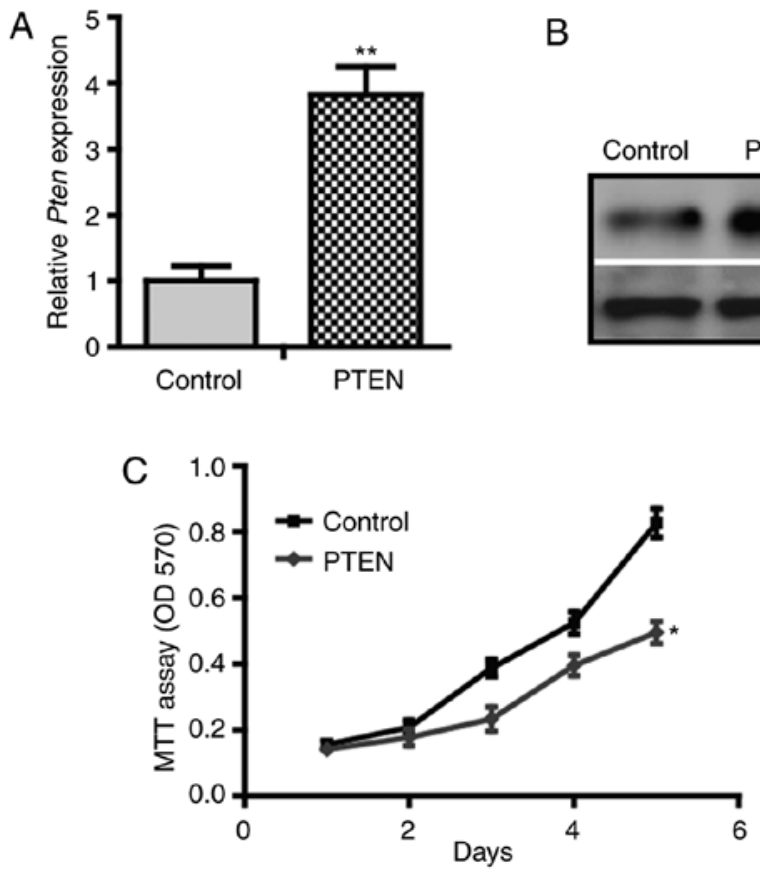

B

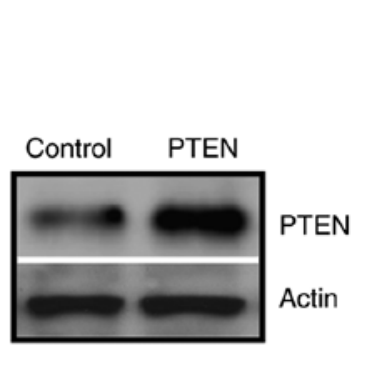

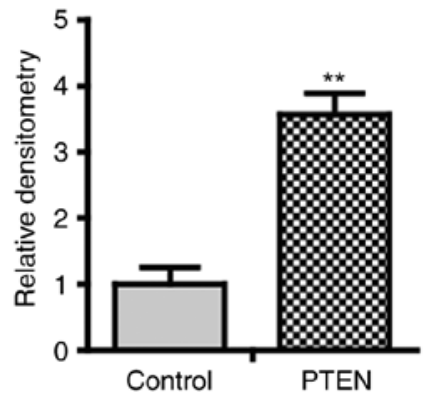

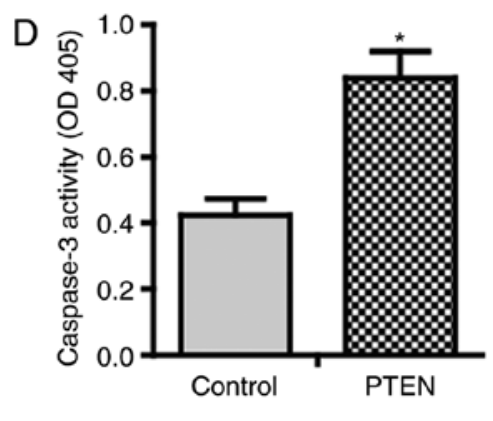

Figure 1. PTEN inhibits proliferation but promotes apoptosis of C3H10T1/2 cells. C3H10T1/2 cells were stably transfected with pcDNA-PTEN or control vectors and were cultured in growth medium. (A) Quantitative PCR for PTEN mRNA expression (n=3). (B) Western blotting for PTEN protein expression. Densitometry of protein bands of PTEN relative to $\beta$-actin, analyzed using ImageJ. Data are representative of 3 independent experiments. (C) MTT assay $(n=6)$. (D) Caspase-3 activity assay $(n=3)$. ${ }^{*} P<0.05 ;{ }^{* *} P<0.01$.

The measurements were performed using BoneJ, a plugin for bone image analysis in ImageJ software (v1.51j8, National Institute of Health).

Statistical analyses. GraphPad Prism software (GraphPad Prism Software, Inc.; version 5.0) was used for statistical analyses. Data are shown as mean \pm SD. The significance of differences was evaluated using the Student's t-test between two groups, or one-way ANOVA among multiple groups, followed by Tukey's post hoc test. $\mathrm{P}<0.05$ was considered to indicate a statistically significant difference.

\section{Results}

PTEN inhibits proliferation but promotes apoptosis of C3H10T1/2 cells. To investigate the role of PTEN in BMP2-induced osteogenic differentiation, a gain-of-function approach was used to overexpress PTEN in C3H10T1/2 cells. Using qPCR analysis, it was demonstrated that transfection with pcDNA-PTEN resulted in a 3.82-fold increase in PTEN mRNA level compared with that of cells transfected with pcDNA3.1 empty vectors (Fig. 1A). Immunoblotting of cell lysate confirmed the induction of PTEN at the protein level (Fig. 1B). Using MTT assay, it was found that PTEN inhibited the proliferation of C3H10T1/2 cells (Fig. 1C). Notably, a higher caspase-3 activity in PTEN-overexpressing cells was observed compared with the control cells (Fig. 1D). These results suggest that PTEN inhibits proliferation but promotes apoptosis of C3H10T1/2 cells, indicating a growth-inhibitory effect.

PTEN inhibits BMP2-induced osteogenic differentiation. Next, the effect of PTEN on BMP2-induced osteogenic differentiation was investigated. Overexpression of PTEN notably inhibited ALP activity by $45 \%$ compared with that of control cells (Fig. 2A). ALP staining exhibited a markedly decreased staining intensity in PTEN-overexpressing cells (Fig. 2B). The expression of osteoblast lineage commitment and early differentiation markers, such as Runx2, osterix (Sp7), ALP ( $A l p l)$, and collagen I (Coll $\alpha 1)$, was downregulated in cells that overexpressed PTEN (Fig. 2C). These data suggest that PTEN inhibits early osteoblast differentiation.

Furthermore, overexpression of PTEN significantly $(\mathrm{P}<0.05)$ decreased the gene expression and secretion of osteocalcin (Fig. 2D and E). The calcium deposition level was decreased in cells that overexpressed PTEN (Fig. 2F). Alizarin red staining showed extensive mineralized nodule formation in control cells. In contrast, the mineralization level was attenuated in cells overexpressing PTEN (Fig. 2G). These results suggest that PTEN inhibits BMP2-induced late osteogenic differentiation, leading to impaired osteoblast maturation characterized by decreased calcium deposition and mineralization.

BMP2 inhibits the expression of PTEN. It was shown that PTEN suppresses BMP2-induced osteogenic differentiation of C3H10T1/2 cells. These observations imply a possible inverse association between BMP2 and PTEN. To test this hypothesis, cells were treated with rhBMP2 at different doses and the gene expression of PTEN was determined.BMP2 decreased the expression of PTEN mRNA in a dose-dependent manner (Fig. 3A). Western blot analysis also revealed an inhibitory effect of BMP2 on the expression of PTEN at the protein level (Fig. 3B and C). These results suggest that BMP2 suppresses the expression of PTEN during osteogenic differentiation.

Activation of PI3K/AKT is required for BMP2 to induce osteogenic differentiation. Next, whether BMP2-mediated inhibition 

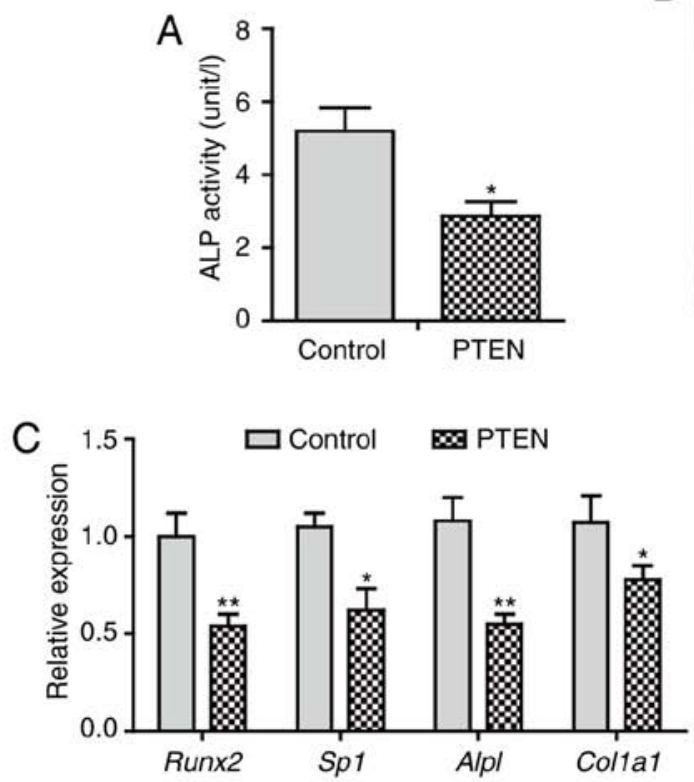

B

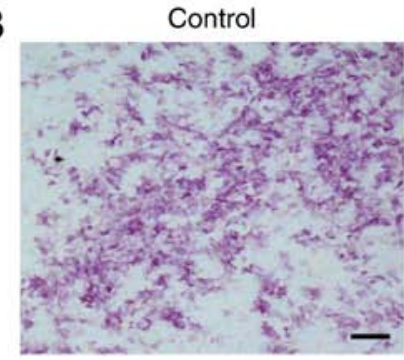

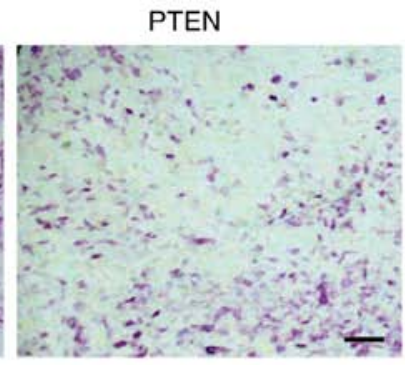

$\mathrm{F}$

Control
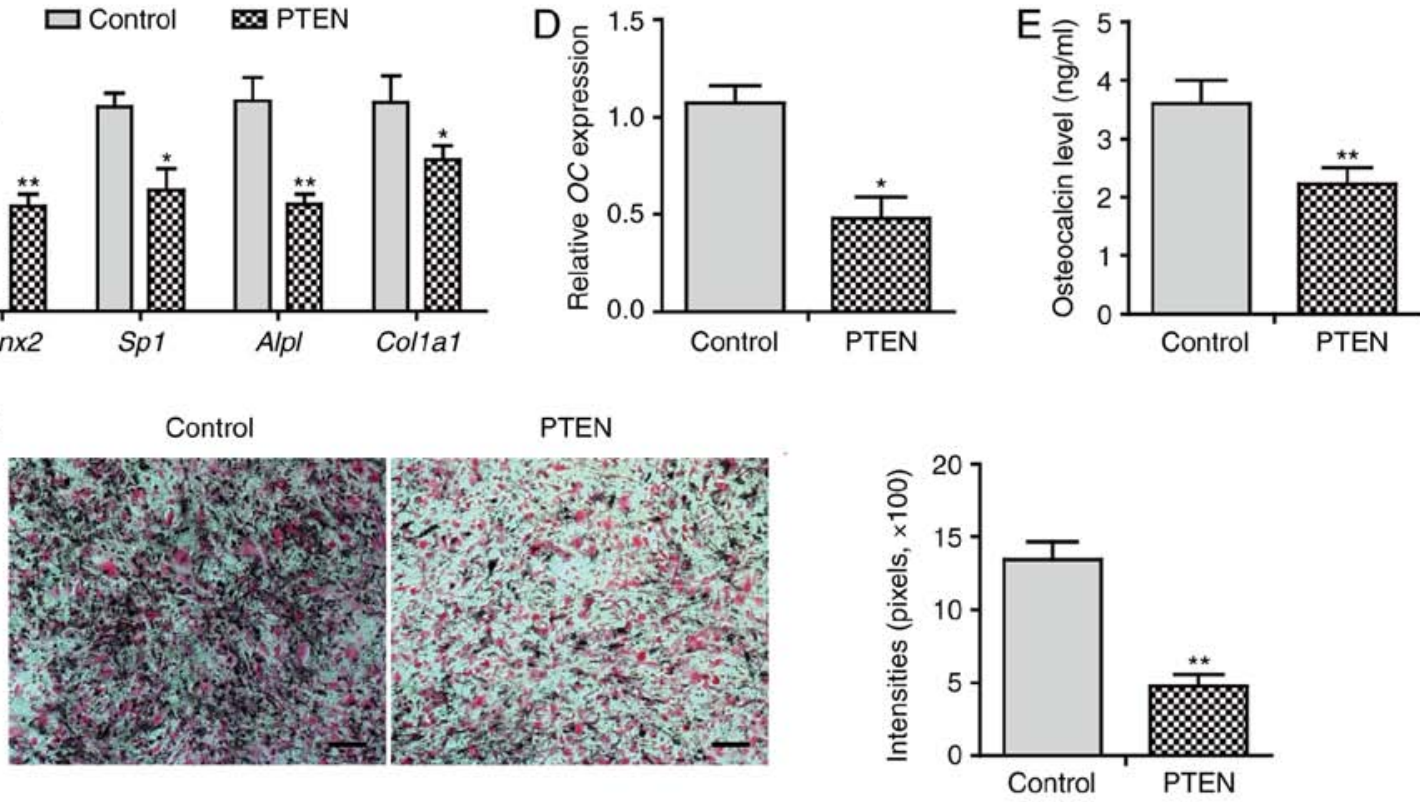

G
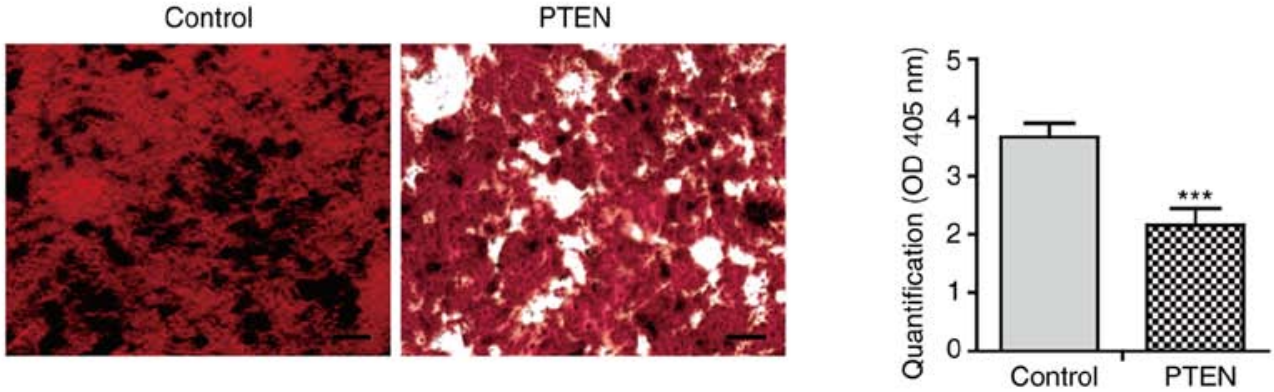

Figure 2. PTEN inhibits BMP2-induced osteogenic differentiation. C3H10T1/2 cells were cultured in BMP2-containing osteogenic medium for indicated periods. (A) ALP activity assay at day $3(n=3)$. (B) ALP staining at day $7(n=3)$. (C) qPCR analysis on day $3(n=3)$. (D) qPCR for OC mRNA at day 5 (n=3). (E) Enzyme-linked immunosorbent assay for osteocalcin secretion in the supernatant at day $7(n=3)$. (F) von Kossa staining in cells cultured in osteogenic medium for 2 weeks. The quantification of stains was performed using ImageJ $(\mathrm{n}=3)$. (G) Alizarin red staining in cells cultured in osteogenic medium for 4 weeks. The quantification of stains was performed using the ARed-Q assay. The sample solution was measured at OD 402 ( $\mathrm{n}=3$ ). Scale bar, $100 \mu \mathrm{m}$. " $\mathrm{P}<0.05$; ${ }^{* *} \mathrm{P}<0.01 ;{ }^{* * * *} \mathrm{P}<0.001$. BMP, bone morphogenetic protein; ALP, alkaline phosphatase; OC, osteocalcin; qPCR, quantitative PCR.

of PTEN could activate PI3K/AKT was investigated. Treatment with rhBMP2 at various doses increased the phosphorylation of AKT in a dose-dependent manner (Fig. 4A), whereas this upregulation was greatly attenuated in the presence of PI3K inhibitor LY294002 (Fig. 4B), suggesting that BMP2 activates the PI3K/AKT pathway. The data support the notion that BMP2 activates PI3K/AKT via its inhibition of PTEN.

It was investigated whether the PI3K/AKT activity is required for BMP2-induced osteogenic differentiation. Cells were cultured in osteogenic medium and treated with PTEN inhibitor bpV(pic) and PI3K inhibitor LY294002. Treatment with $\mathrm{bpV}(\mathrm{pic})$ increased ALP activity. In contrast, the addition of LY294002 decreased both baseline and bpV(pic)-induced activity of ALP (Fig. 4C). Similarly, the secretion of osteocalcin was increased in the presence of $\mathrm{bpV}(\mathrm{pic})$. However, this induction was strongly reversed by LY294002 (Fig. 4D). These results confirm that PTEN acts as a suppressor for osteoblastogenesis and, more importantly, support the idea that activation of the PI3K/AKT is indispensable for BMP2 to induce osteogenic differentiation.

Inactivation of the PI3K/AKT pathway suppresses BMP2-induced ectopic bone formation. Further, the role of PI3K/AKT during in vivo ectopic bone formation was 


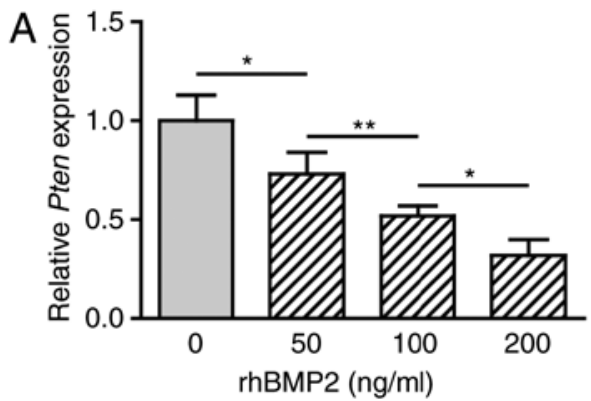

B

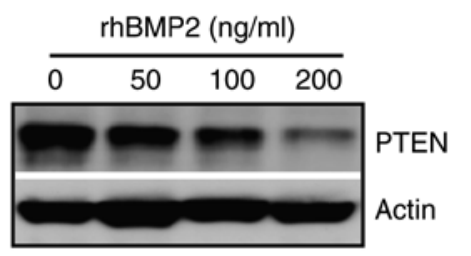

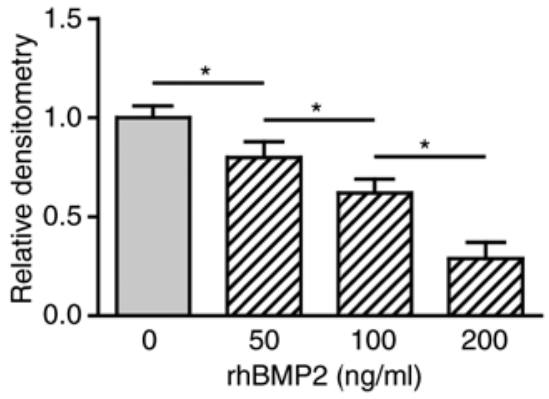

Figure 3. BMP2 inhibits PTEN gene expression. C3H10T1/2 cells were cultured in osteogenic medium containing rhBMP2 at various doses $(0,50,100$ and $200 \mathrm{ng} / \mathrm{ml}$ ) for $48 \mathrm{~h}$. (A) Quantitative PCR for PTEN mRNA (n=3). (B) Western blotting for PTEN expression. Densitometry for the protein band of PTEN relative to $\beta$-actin, analyzed using ImageJ. Data are representative of 3 independent experiments. ${ }^{*} \mathrm{P}<0.05 ;{ }^{* *} \mathrm{P}<0.01$. BMP, bone morphogenetic protein, rhBMP2, recombinant human BMP2.

A

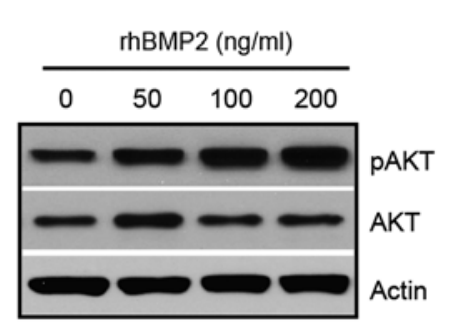

B

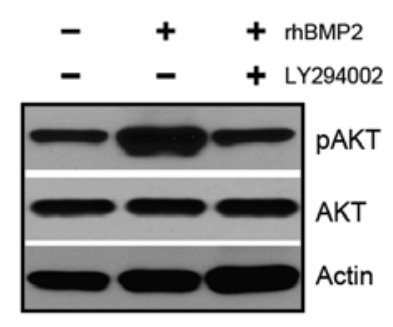

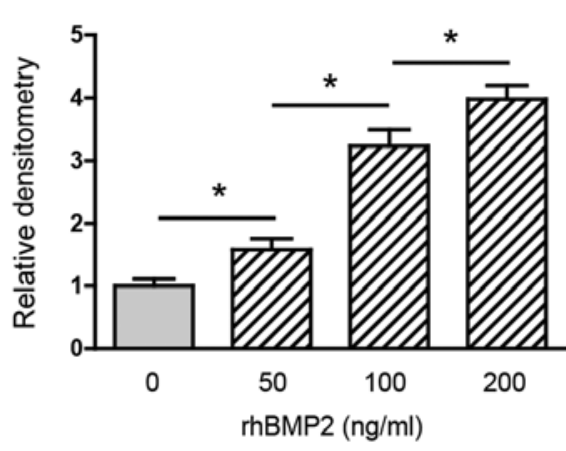

C
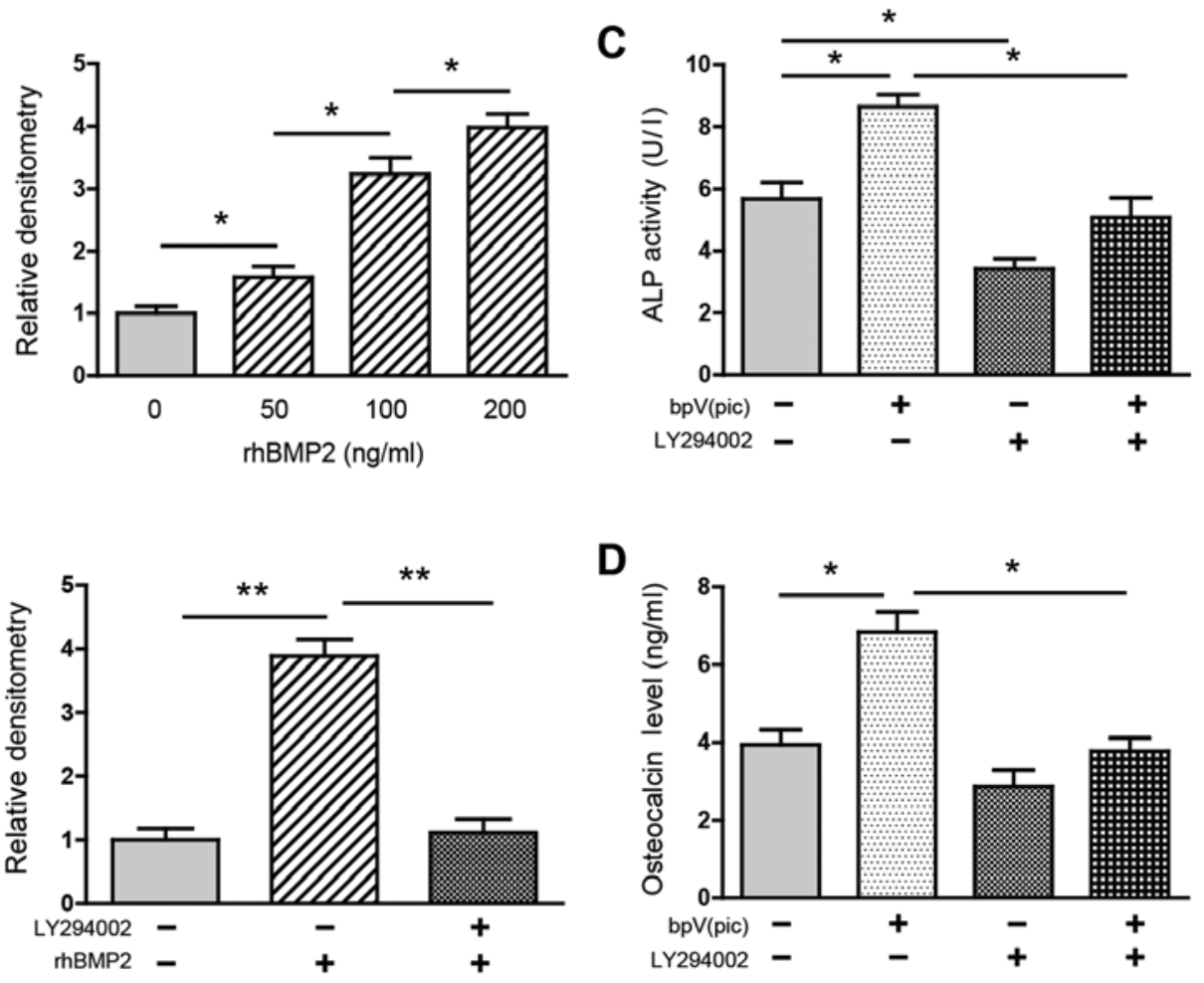

D

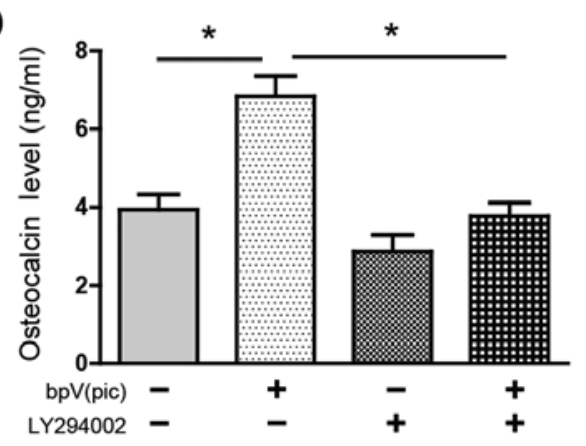

Figure 4. Activation of PI3K/AKT is required for BMP2-induced osteogenic differentiation. (A) C3H10T1/2 cells were cultured in osteogenic medium containing BMP2 at various doses $(0,50,100$ or $200 \mathrm{ng} / \mathrm{ml})$ for $48 \mathrm{~h}$. The expression of pAKT and total AKT was examined using western blotting. Densitometry for the ratio of pAKT/AKT protein bands was analyzed using ImageJ. Data are representative of 3 independent experiments. (B) C3H10T1/2 cells were cultured in osteogenic medium containing BMP2 $(0$ and $200 \mathrm{ng} / \mathrm{ml})$ with/without LY294002 $(10 \mu \mathrm{M})$ for $48 \mathrm{~h}$. The expression of pAKT and AKT was examined using western blotting. Densitometry for the ratio of pAKT/AKT protein bands was analyzed using ImageJ. Data are representative of 3 independent experiments. (C) ALP activity assay in C3H10T1/2 cells cultured in BMP2 (200 ng/ml)-containing osteogenic medium, treated with bpV(pic) (10 $\mu \mathrm{M})$ and/or LY294002 $(10 \mu \mathrm{M})$ for 3 days $(\mathrm{n}=3)$. (D) Enzyme-linked immunosorbent assay for osteocalcin secretion in supernatant harvested from C3H10T1/2 cells cultured in osteogenic medium for 7 days $(\mathrm{n}=3)$. ${ }^{*} \mathrm{P}<0.05 ;{ }^{* *} \mathrm{P}<0.01$. BMP, bone morphogenetic protein; pAKT, phosphorylated AKT; ALP, alkaline phosphatase.

elucidated. Nine days after the operation, implantation of rhBMP2 induced the formation of a large area of a cartilaginous matrix in control mice, as indicated by the positive red stain with Safranin O. Strikingly, animals treated with LY294002 exhibited markedly decreased chondrogenic differentiation at this time point, as characterized by a substantially decreased area of the cartilaginous matrix (Fig. 5A and Di). At 3 weeks post-operation, X-ray radiography in control mice detected the formation of ectopic new bone. However, only a mildly ossified area was present in mice treated with LY294002 (Fig. 5B). Histological analysis revealed the development of mature bone tissue, which was characterized by a well-defined perimeter of the cortical bone rim, in which a large number of trabeculae and a marrow cavity containing bone marrow and adipocyte-like cells were identified. Osteoblasts along the trabeculae or osteocytes embedded within the bone were also observed. Notably, mice treated with LY294002 displayed highly decreased bone formation, in that only a small number of trabeculae had developed, and the bone marrow cavity was mainly filled with a large area of adipocyte-like cells with only a few bone marrow cells present (Fig. 5C). The bone histomorphometric analysis further indicated that LY294002-treated animals exhibited 
A

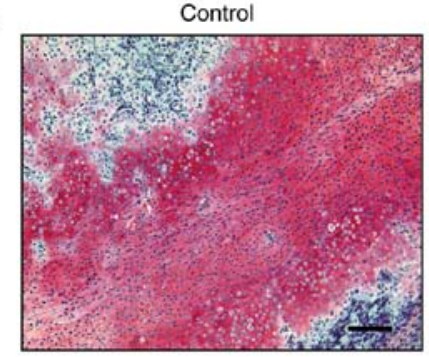

C

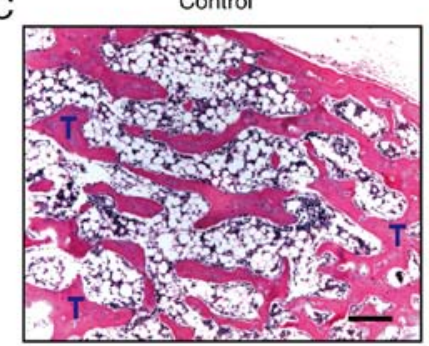

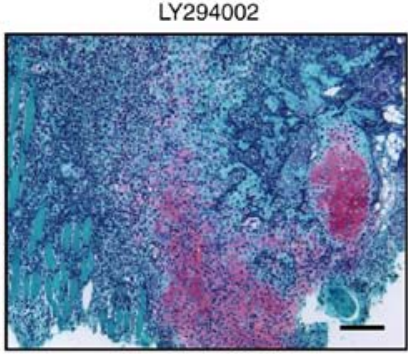

LY294002

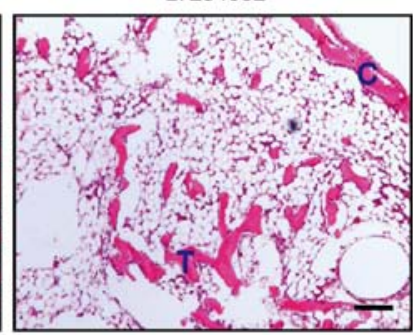

B

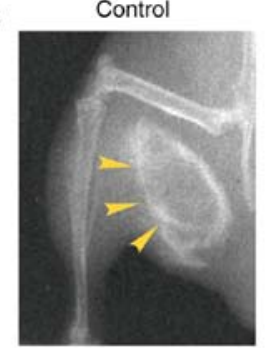

LY294002

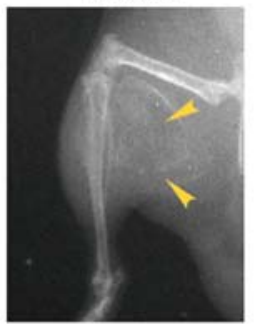

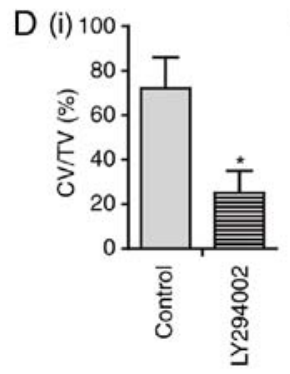
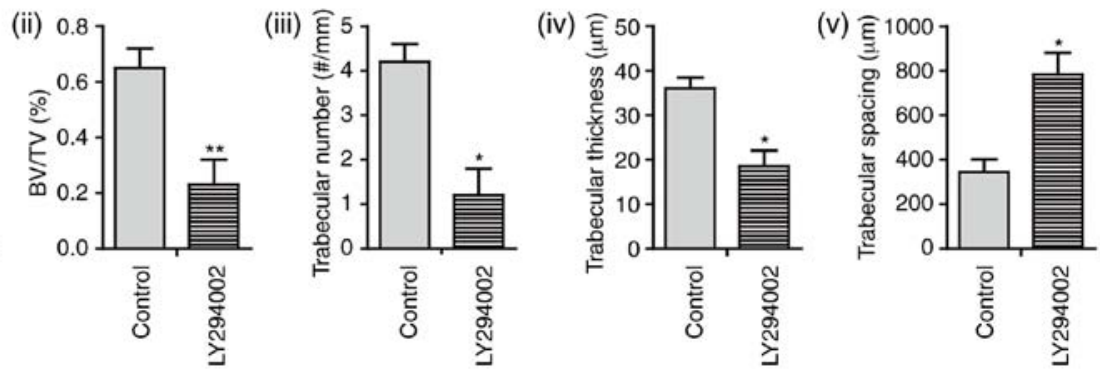

Figure 5. Inactivation of PI3K/AKT suppresses BMP2-induced endochondral ossification. (A) Safranin O staining at 9 days after the operation. (B) X-ray radiography for bone formation at 3 weeks after the operation. (C) Hematoxylin and eosin staining at 3 weeks after the operation. (D) Bone histomorphometric analysis, including i) the ratio of $\mathrm{CV} / \mathrm{TV} \%$, ii) the BV/TV\%, iii) trabecular number, iv) trabecular thickness and v) trabecular spacing (n=3). Scale bar, $100 \mu \mathrm{m}$. ${ }^{*} \mathrm{P}<0.05 ;{ }^{* *} \mathrm{P}<0.01$. BMP, bone morphogenetic protein; $\mathrm{C}$, cortical rim; $\mathrm{T}$, trabecular structure; CV/TV, cartilage volume/tissue volume; $\mathrm{BV} / \mathrm{TV}$, ratio of bone volume/tissue volume.

a decreased ratio of bone volume/tissue volume (BV/TV), decreased trabecular number, and decreased trabecular thickness, but increased trabecular spacing, compared with those of control mice (Fig. 5Dii-v). These results suggest that treatment with LY294002 dramatically suppresses intramuscular endochondral ossification, supporting the notion that the activity of the PI3K/AKT pathway is critically implicated in BMP2-induced ectopic bone formation.

\section{Discussion}

The present study reports that PTEN inhibits BMP2-induced osteoblast differentiation, whereas BMP2 represses PTEN expression, leading to the activation of PI3K/AKT. In vivo, the PI3K inhibitor suppresses BMP2-induced intramuscular bone formation. The present results suggest an inverse association between PTEN and BMP2, and also support the notion that $\mathrm{PI} 3 \mathrm{~K} / \mathrm{AKT}$ signaling activity is indispensable for BMP2 to induce ectopic ossification. Hence, the present findings highlight a critical implication of the PTEN/PI3K/AKT pathway during the pathogenesis of $\mathrm{HO}$.

Since BMP2 can drive the expression of Runx 2 and osterix and functions as a key regulator during osteoblast differentiation $(18,19)$, BMP2 was used as an osteogenic agent to induce osteoblast differentiation in vitro and ectopic bone formation in vivo. BMP2-induced osteoblastogenesis has been widely reported in a variety of cell types, such as mesenchymal stem cells, osteoblast precursors and osteoblasts $(12,20,21)$. BMP2 also associates with the pathogenesis of $\mathrm{HO}(2,3)$. Implantation of recombinant BMP 2 has been a standard procedure to induce endochondral bone formation in soft tissues, including muscle, ligament and subcutaneous tissue (22-24).

The involvement of the PTEN/PI3K/AKT pathway in skeletal development and bone repair has been previously investigated. However, the role of this pathway nexus in $\mathrm{HO}$ remains unknown. In the present study, PTEN was overexpressed in MSC-like C3H10T1/2 cells and this tumor suppressor inhibited proliferation but enhanced apoptosis, indicating a growth-inhibitory effect. This result is not surprising, in that PTEN has been documented to promote proapoptotic activity in a variety of cancer and noncancerous cells, including MSCs and osteoblasts $(6,25)$. Interestingly, it was further reported that PTEN suppressed BMP2-induced osteoblast differentiation, including the commitment of osteoblast lineage, early and late osteoblast differentiation, and osteoblast mineralization. The present results appear in agreement with a previous study, in which osteoblasts deficient in the PTEN gene displayed increased expression of several osteoblast markers, including Runx2, ALP, collagen I and osteocalcin (6). Based on these findings, it seems highly plausible that PTEN functions as a 
suppressor for osteoblast differentiation by inhibiting cell growth and the entire differentiation process.

Given that PTEN is a suppressor during BMP2-triggered osteogenic differentiation, it was deduced that an inverse association might exist between BMP2 and PTEN. Thus far, the association between these two molecules seems controversial. Waite and Eng (26) reported that exposure to BMP2 increases PTEN protein expression in MCF7 human breast cancer cells, yet this induction is rapid and attributed to the inhibition of PTEN degradation rather than an increase in protein synthesis. In contrast, Beck and Carethers (11) showed that BMP2 downregulates PTEN expression in SW480 human colon cancer cells, an effect mediated via the RAS/ERK signaling pathway. This discrepancy may be due to different time lengths of BMP2 treatment used in these studies or different cell types. The BMP2-mediated rapid increase in PTEN protein occurred within $6 \mathrm{~h}$ in response to BMP2, peaking at $1 \mathrm{~h}(26)$, whereas BMP2 resulted in a reduction of PTEN after $36 \mathrm{~h}$ of BMP2 treatment, and this inhibitory effect could last at least 7 days (11). The present study reported that BMP2 decreased PTEN expression at mRNA and protein levels in C3H10T1/2 cells cultured in osteogenic medium for $48 \mathrm{~h}$. As such, the present results suggest a negative association between BMP2 and PTEN and also support the notion that BMP2 exerts its osteogenic differentiation capacity via its negative regulation of PTEN.

The present study showed that BMP2-mediated downregulation of PTEN was closely associated with activation of $\mathrm{PI} 3 \mathrm{~K} / \mathrm{AKT}$, in that BMP2 increased the level of AKT phosphorylation. However, this upregulation could be markedly attenuated by the PI3K inhibitor LY294002. Functionally it was further demonstrated that the PTEN inhibitor bpV(pic) increased the activity of ALP and the secretion of osteocalcin, the two important markers for early and late osteoblast differentiation, respectively. Notably, bpV(pic)-induced upregulation of ALP and osteocalcin was substantially reversed by LY294002. Hence, the present results indicate that the PI3K/AKT signaling activity is indispensable for BMP2 to induce osteogenic differentiation. In support of the present study, Ghosh-Choudhury et al (10) reported that the expression of a dominant-negative PI3K in 2T3 osteoblast precursor cells abolishes the induction of ALP in response to BMP2, indicating the requirement of PI3K for BMP2-induced osteoblastogenesis. Mukherjee and Rotwein (12) also reported that a dominant-negative AKT is potent in preventing osteoblast differentiation by BMP2. Therefore, results from the present study and others suggest that activation of PI3K/AKT plays a crucial role during BMP2-induced osteogenic differentiation.

Importantly, it was reported that the inactivation of PI3K/AKT dramatically inhibited ectopic bone formation. In the present study, implantation of the recombinant BMP2 into muscle induced chondrogenic differentiation 9 days after the operation, as evidenced by the formation of a large area of cartilaginous matrix. However, treatment with PI3K inhibitor induced only mild cartilage deposition. This effect suggests that the antagonization of PI3K/AKT decreases chondrogenesis. These findings are consistent with Iwasa et al (27), who reported that the knockdown of PTEN in adult human chondrocytes activates PI3K/AKT and increases the expression and synthesis of chondrocyte markers, including collagen II, aggrecan and proteoglycan.
Furthermore, the implantation of BMP2 induced a well-defined mature bone structure 3 weeks after the operation. In contrast, treatment with LY294002 greatly suppressed new bone development, suggesting that BMP2-induced bone-forming capacity is impaired by the inactivation of PI3K/AKT. In support of the present results, Ford-Hutchinson et al (28) reported that a collagen II (COL2Al) promoter-driven PTEN gene deletion could activate the PI3K signaling and induce a dramatic increase in the amount of trabecular and cortical bone during postnatal bone development. Therefore, the current findings support the idea that $\mathrm{PI} 3 \mathrm{~K} / \mathrm{AKT}$ activity is critically required during BMP2-induced ectopic endochondral bone formation.

The results of the present study may have significant clinical relevance. $\mathrm{HO}$ is defined as the formation of extraskeletal bone in soft tissues (such as muscle and ligament), and it may cause severe clinical problems. For example, the ossification of the OPLL of the cervical spine can lead to serious myelopathy due to compression of the spinal cord and nerve roots (29), whereas $\mathrm{HO}$ following arthroplasty can become symptomatic and result in functional disability (30). Unfortunately, there is currently a lack of effective drug therapy against HO. The PI3K/AKT pathway has been an attractive target for cancer therapy (31). It is proposed that targeting PI3K/AKT using small-molecule inhibitor(s) may also be seriously considered for the treatment of $\mathrm{HO}$.

In general, the present study reports that PTEN inhibits proliferation but promotes apoptosis of mesenchymal pluripotent C3H10T1/2 cells, and inhibits BMP2-induced osteogenic differentiation. On the other hand, BMP2 represses PTEN, leading to the activation of PI3K/AKT, which is indispensable for BMP2 to induce osteoblastogenesis. Inactivation of the PI3K/AKT pathway markedly suppresses BMP2-induced endochondral ossification in muscles. The present results demonstrate for the first time that the PTEN/PI3K/AKT signaling nexus is critically involved in the development of ectopic bone formation. Targeting the PI3K/AKT pathway using inhibitor(s) may represent a potential molecular therapy against $\mathrm{HO}$.

\section{Acknowledgements}

Not applicable.

\section{Funding}

This study was supported by the Shandong Provincial Medical Technology Development Project to J.D. (grant no. 2019WS459).

\section{Availability of data and materials}

The datasets used and/or analyzed during the current study are available from the corresponding author on reasonable request.

\section{Authors' contributions}

JD and BT conceived the study and wrote the manuscript. JD, XX, QZ and ZY performed experiments and contributed data. JD, XX, QZ and BT performed data analysis. All authors approved the final version of the manuscript. 


\section{Ethics approval and consent to participate}

All animal procedures were reviewed and approved by the Institutional Animal Care and Use Committee of the Shandong Provincial Hospital Affiliated to Shandong First Medical University (approval no. M2018143).

\section{Patient consent for publication}

Not applicable.

\section{Competing interests}

The authors declare that they have no competing interests.

\section{References}

1. Scott MA, Levi B, Askarinam A, Nguyen A, Rackohn T, Ting K, Soo $\mathrm{C}$ and James AW: Brief review of models of ectopic bone formation. Stem Cells Dev 21: 655-667, 2012.

2. Łegosz P, Drela K, Pulik Ł, Sarzyńska S and Małdyk P. Challenges of heterotopic ossification-molecular background and current treatment strategies. Clin Exp Pharmacol Physiol 45: 1229-1235, 2018

3. Shi L, Sun W, Gao F, Cheng L and Li Z: Heterotopic ossification related to the use of recombinant human BMP-2 in osteonecrosis of femoral head. Medicine (Baltimore) 96: e7413, 2017.

4. PulidoR: PTEN inhibition in human disease therapy. Molecules 23: 285,2018

5. Papa A and Pandolfi PP: The PTEN-PI3K axis in cancer. Biomolecules 9: 153, 2019.

6. Liu X, Bruxvoort KJ,Zylstra CR, Liu J, Cichowski R, Faugere MC, Bouxsein ML, Wan C, Williams BO and Clemens TL: Lifelong accumulation of bone in mice lacking Pten in osteoblasts. Proc Natl Acad Sci USA 104: 2259-2264, 2007.

7. Burgers TA, Hoffmann MF, Collins CJ, Zahatnansky J, Alvarado MA, Morris MR, Sietsema DL, Mason JJ, Jones CB Ploeg HL, et al: Mice lacking pten in osteoblasts have improved intramembranous and late endochondral fracture healing. PLoS One 8: e63857, 2013.

8. Xiong Y, Cao F, Hu L, Yan C, Chen L, Panayi AC, Sun Y, Zhou W, Zhang $\mathrm{P}, \mathrm{Wu} \mathrm{Q}$, et al: miRNA-26a-5p accelerates healing via downregulation of PTEN in fracture patients with traumatic brain injury. Mol Ther Nucleic Acids 17: 223-234, 2019.

9. Kawamura N, Kugimiya F, Oshima Y, Ohba S, Ikeda T, Saito T, Shinoda Y, Kawasaki Y, Ogata N, Hoshi K, et al: Akt1 in osteoblasts and osteoclasts controls bone remodeling. PLoS One 2: e1058, 2007.

10. Ghosh-Choudhury N, Abboud SL, Nishimura R, Celeste A, Mahimainathan L and Choudhury GG: Requirement of BMP-2-induced phosphatidylinositol 3-kinase and Akt serine/ threonine kinase in osteoblast differentiation and Smad-dependent BMP-2 gene transcription. J Biol Chem 277: 33361-33368, 2002.

11. Beck SE and Carethers JM: BMP suppresses PTEN expression via RAS/ERK signaling. Cancer Biol Ther 6: 1313-1317, 2007.

12. Mukherjee A and Rotwein P: Akt promotes BMP2-mediated osteoblast differentiation and bone development. J Cell Sci 122: 716-726, 2009.

13. Mukherjee A, Wilson EM and Rotwein P: Selective signaling by Akt 2 promotes bone morphogenetic protein 2-mediated osteoblast differentiation. Mol Cell Biol 30: 1018-1027, 2010.

14. Zhao L, Li G, Chan KM, Wang Y and Tang PF: Comparison of multipotent differentiation potentials of murine primary bone marrow stromal cells and mesenchymal stem cell line C3H10T1/2. Calcif Tissue Int 84: 56-64, 2009.
15. Lee CH, Jin MU, Jung HM, Lee JT and Kwon TG: Effect of dual treatment with SDF-1 and BMP-2 on ectopic and orthotopic bone formation. PLoS One 10: e0120051, 2015.

16. Livak KJ and Schmittgen TD: Analysis of relative gene expression data using real-time quantitative PCR and the 2(-Delta Delta C(T)) method. Methods 25: 402-408, 2001.

17. Egan KP, Brennan TA and Pignolo RJ: Bone histomorphometry using free and commonly available software. Histopathology 61: 1168-1173, 2012

18. Matsubara T, Kida K, Yamaguchi A, Hata K, Ichida F, Meguro H, Aburatani H, Nishimura R and Yoneda T: BMP2 regulates Osterix through Msx2 and Runx2 during osteoblast differentiation. J Biol Chem 283: 29119-29125, 2008.

19. Lee MH, Kim YJ, Kim HJ, Park HD, Kang AR, Kyung HM, Sung JH, Wozney JM, Kim HJ and Ryoo HM: BMP-2-induced Runx 2 expression is mediated by Dlx5, and TGF-beta 1 opposes the BMP-2-induced osteoblast differentiation by suppression of Dlx5 expression. J Biol Chem 278: 34387-34394, 2003.

20. Bain G, Müller T, Wang X and Papkoff J: Activated beta-catenin induces osteoblast differentiation of $\mathrm{C} 3 \mathrm{H} 10 \mathrm{~T} 1 / 2$ cells and participates in BMP2 mediated signal transduction. Biochem Biophys Res Commun 301: 84-91, 2003.

21. Ogasawara T, Kawaguchi H, Jinno S, Hoshi K, Itaka K, Takato T, Nakamura $\mathrm{K}$ and Okayama $\mathrm{H}$ : Bone morphogenetic protein 2 -induced osteoblast differentiation requires Smad-mediated down-regulation of Cdk6. Mol Cell Biol 24: 6560-6568, 2004.

22. Hashimoto K, Kaito T, Furuya M, Seno S, Okuzaki D, Kikuta J, Tsukazaki $\mathrm{H}$, Matsuda $\mathrm{H}$, Yoshikawa $\mathrm{H}$ and Ishii $\mathrm{M}$ : In vivo dynamic analysis of BMP-2-induced ectopic bone formation. Sci Rep 10: 4751, 2020.

23. Chen Y, Whetstone HC, Youn A, Nadesan P, Chow EC, Lin AC and Alman BA: Beta-catenin signaling pathway is crucial for bone morphogenetic protein 2 to induce new bone formation. J Biol Chem 282: 526-533, 2007.

24. Hoshi K, Amizuka N, Sakou T, Kurokawa T and Ozawa H: Fibroblasts of spinal ligaments pathologically differentiate into chondrocytes induced by recombinant human bone morphogenetic protein-2: Morphological examinations for ossification of spinal ligaments. Bone 21: 155-162, 1997.

25. Yamada KM and Araki M: Tumor suppressor PTEN: Modulator of cell signaling, growth, migration and apoptosis. J Cell Sci 114: 2375-2382, 2001.

26. Waite KA and Eng C: BMP2 exposure results in decreased PTEN protein degradation and increased PTEN levels. Hum Mol Genet 12: 679-684, 2003.

27. Iwasa K, Hayashi S, Fujishiro T, Kanzaki N, Hashimoto S, Sakata S, Chinzei N, Nishiyama T, Kuroda R and Kurosaka M: PTEN regulates matrix synthesis in adult human chondrocytes under oxidative stress. J Orthop Res 32: 231-237, 2014

28. Ford-Hutchinson AF, Ali Z, Lines SE, Hallgrimsson B, Boyd SK and Jirik FR: Inactivation of Pten in osteo-chondroprogenitor cells leads to epiphyseal growth plate abnormalities and skeletal overgrowth. J Bone Miner Res 22: 1245-1259, 2007.

29. Yan L, Gao R, Liu Y, He B, Lv S and Hao D: The pathogenesis of ossification of the posterior longitudinal ligament. Aging Dis 8 : 570-582, 2017.

30. Biz C, Pavan D, Frizziero A, Baban A and Iacobellis C: Heterotopic ossification following hip arthroplasty: A comparative radiographic study about its development with the use of three different kinds of implants. J Orthop Surg Res 10: 176, 2015.

31. Yang J, Nie J, Ma X, Wei Y, Peng Y and Wei X: Targeting PI3K in cancer: Mechanisms and advances in clinical trials. Mol Cancer 18: 26, 2019.

(7) $(-)$ This work is licensed under a Creative Commons Attribution-NonCommercial-NoDerivatives 4.0 International (CC BY-NC-ND 4.0) License. 